\section{Inflaming resistance to Tarceva}

\section{By Chris Cain, Staff Writer}

A team at Cold Spring Harbor Laboratory has found that in cancer, inflammation-driven IL-6 signaling can cause resistance to drugs that inhibit epidermal growth factor receptor, such as Tarceva erlotinib and Iressa gefitinib. ${ }^{1}$ The data suggest that blocking IL- 6 could help treat drugresistant cancers, a hypothesis Alder Biopharmaceuticals Inc. may put to the test in a Phase IIa trial of its anti-IL-6 antibody, ALD518.

About $80 \%$ of patients who respond to small molecule inhibitors of epidermal growth factor receptor (EGFR) carry oncogenic mutations in the kinase domain of the receptor that render them sensitive to EGFR inhibition. ${ }^{2}$ However, all patients will eventually develop resistance to these drugs.

Half of resistant cases stem from secondary mutations within EGFR or amplification of c-Met proto-oncogene (MET; HGFR). In the other half of cases, the mechanisms underlying resistance are unknown.

Thus, a team led by Raffaella Sordella, assistant professor at Cold Spring Harbor Laboratory, sought to identify new mechanisms of drug resistance. The researchers started by selecting EGFR mutant cell lines that were resistant to Tarceva but lacked any of the known mutations that confer resistance.

"We noticed these erlotinib-resistant cells had a dramatically different morphology than erlotinib-sensitive cells," Sordella told SciBX. "We performed gene expression profiling to understand what was different about them."

Her team found that transforming growth factor- $\beta$ (TGFB; TGF $\beta$ ) was induced in resistant cells, explaining their difference in morphology. Moreover, IL-6 secretion from these cells was induced more than 10 -fold, and the increase required TGF $\beta$.

Adding IL-6 to erlotinib-sensitive cells increased their resistance to the drug compared with that of cells not given IL-6.

Sordella's team also examined non-small cell lung cancer (NSCLC) samples isolated from Tarceva-naïve patients and found that a subpopulation of cells had high levels of TGF $\beta$ and IL-6. That finding suggests some tumor cells may be intrinsically resistant to Tarceva.

Tarceva is marketed by Astellas Pharma Inc. and Roche's Genentech Inc. unit, whereas Iressa is marketed by AstraZeneca plc.

\section{No escape}

"These experiments demonstrate that high levels of IL- 6 driven by TGF $\beta$ can release cancer cells from their dependency on EGFR signaling," said Sordella.
Alder may put that mechanism to the test. "We are strongly considering running a Phase IIa trial examining the effect of combination therapy using ALD518 and Tarceva," Mark Litton, CBO and cofounder, told SciBX. "It is very timely that this paper has come out suggesting that this could be beneficial."

"By only targeting EGFR, you select for the survival of cells that express high levels of IL-6 independent of EGFR signaling," noted Jeffrey Smith, CMO and cofounder of Alder. "If you block both IL- 6 and EGFR, you stop this escape mechanism."

Smith said the Phase IIa trial is in the planning stages and will compare the efficacy of Tarceva alone with that of ALD518 plus Tarceva. In addition to the standard endpoints such as response rate, progressionfree survival (PFS) and overall survival (OS), they plan to do molecular phenotyping to look at the response in more detail to aid in the design of possible Phase IIb/III studies.

The biotech already has tested ALD518 as a stand-alone therapy. In June, the company announced preliminary data from a Phase IIa trial of the antibody to treat cachexia and anemia in late-stage NSCLC patients. Patients receiving ALD518 had higher hemoglobin levels and less weight loss than patients given placebo.

\section{Other options}

The results from the Cold Spring Harbor group, which were published in the Proceedings of the National Academy of Sciences, build on previous studies that illustrated IL-6's role in EGFR mutant lung cancers.

In 2007, a group led by Jacqueline Bromberg, associate professor of medicine at Memorial Sloan-Kettering Cancer Center, showed that EGFR mutant NSCLC samples expressed high levels of IL-6. Inhibiting IL- 6 or its downstream signaling partners, including Janus kinase (JAK), stopped cancer growth in xenograft mice carrying NSCLC cells ${ }^{3}$ (see Figure 1, “Targeting Tarceva resistance”).

Bromberg told SciBX that the new PNAS paper "advances the field by demonstrating a link between TGF $\beta$ and IL- 6 signaling. It suggests that a combined approach blocking both EGFR and JAK/STAT signaling could be beneficial for cancer treatment."

Blocking this pathway could be broadly beneficial in EGFR mutant cancer. In addition to its role in NSCLC, a recent study suggests the JAK and signal transducer and activator of transcription (STAT) pathway may be a therapeutic target in EGFR mutant glioblastoma multiforme ${ }^{4}$ (see Box 1, “Targeting IL-6 in glioblastoma”).

Incyte Corp.'s INCB18424, the most advanced JAK-1 and JAK-2 inhibitor, is in Phase III testing to treat myelofibrosis. Incyte declined to comment for this article. At least six other companies have compounds in development to block IL-6 and JAK signaling in cancer and autoimmune disease.

In addition to specifically targeting the IL-6 pathway, Sordella suggested that generally targeting inflammation, for example with a cyclooxygenase-2 (COX-2) inhibitor, could help treat Tarceva-resistant cancers.

"Inflammation involves the recruitment of immune cells, which can ultimately lead to increased levels of IL-6," she said. "It is important 
Figure 1. Targeting Tarceva resistance. Yao et al. suggest that blocking IL-6 signaling could reduce resistance to epidermal growth factor receptor (EGFR) inhibitors like Tarceva erlotinib. In non-small cell lung cancer (NSCLC), mutations in the kinase domain of EGFR [a] lead to increased production and secretion of IL-6. IL-6 signals through gp130, Janus kinase-1 (JAK-1) and JAK-2 to increase phosphorylation $(P)$ of signal transducer and activator of transcription 3 (STAT3), a prosurvival transcription factor [b]. Thus, by blocking EGFR, a tumor should be rendered unable to progress. But in Tarceva-resistant tumors, transforming growth factor- $\beta$ (TGFB; TGF $\beta$ ) upregulates IL-6 independent of EGFR signaling [c].

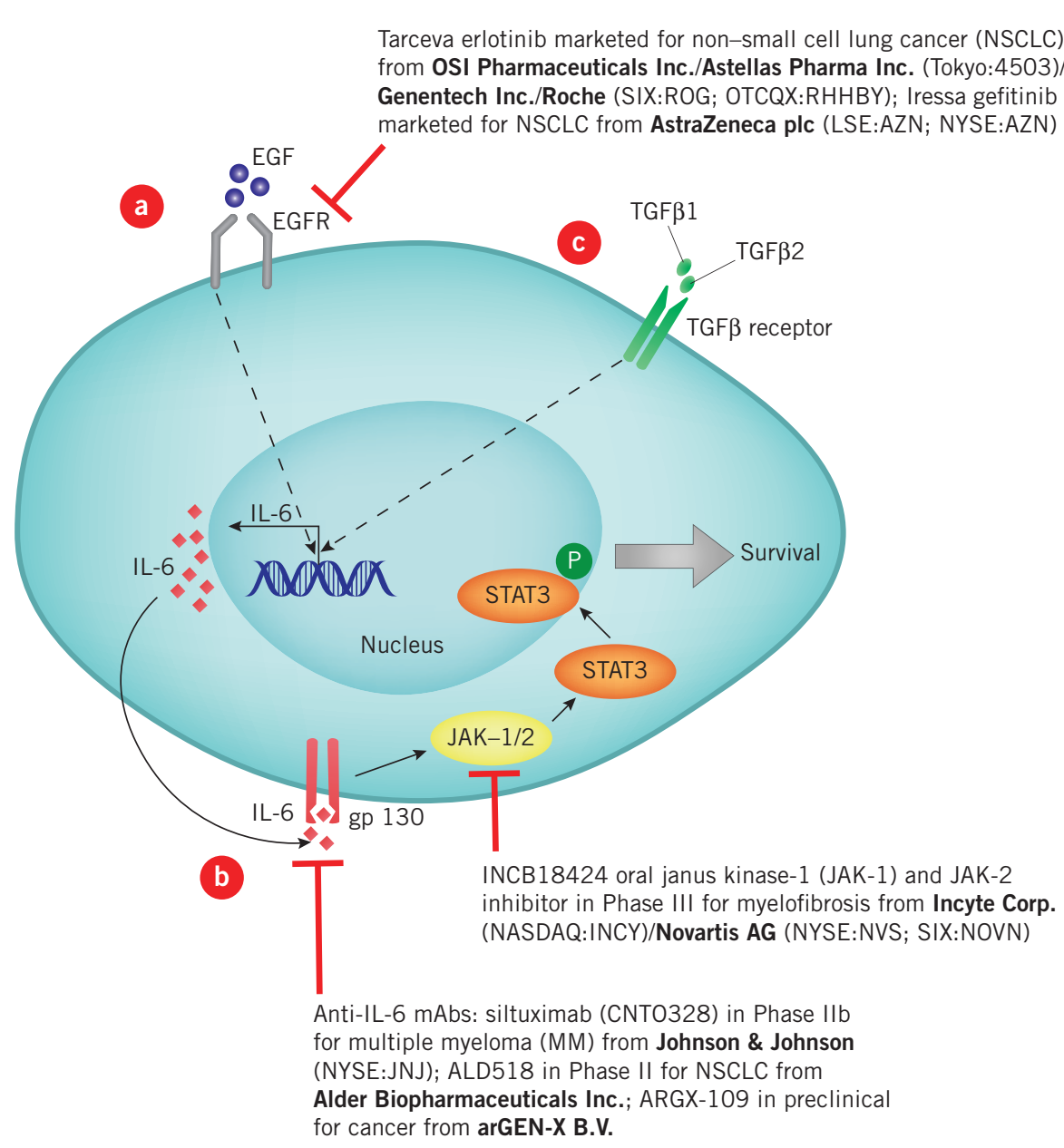

to understand the influence of the tumor microenvironment on drug sensitivity."

For example, her team reported in the PNAS paper that lipopolysaccharide (LPS), a general stimulator of inflammation, induced IL-6 expression in mice and reduced NSCLC sensitivity to Tarceva compared with no treatment.

Kevin Struhl, professor of biological chemistry and molecular pharmacology at Harvard Medical School, also argued for a broad attack against inflammation. "My view is that the inflammatory pathway is acting as a general stress response in many cell types," he told SciBX.

Struhl's lab has shown in cell culture studies that IL-6 can trigger a positive feedback loop that maintains cancer cell survival even in the absence of an initial oncogenic signal. In a paper published last month in Molecular Cell, members of his lab identified microRNA-21 (miR21) as a key component of that positive feedback loop. ${ }^{5}$

miR-21 is upregulated in EGFR mutant NSCLC, and combined inhibition of miR-21 and EGFR triggered increased apoptosis in an NSCLC cell line. ${ }^{6}$

\section{Next steps}

Regardless of whether inflammation is broadly dampened or specifically decreased via blocking IL-6, both Sordella and Bromberg said the key question is whether lower levels of inflammation could help treat EGFR-driven NSCLC in humans.

Bromberg is studying the effect of JAK inhibitors in mice with NSCLC. "So far our studies have shown blocking IL-6 signaling can stop tumor progression but does not cause tumor regression," she said. "We still don't know if targeting this pathway will be effective in treating disease."

Sordella plans to continue studying the therapeutic effects of combination therapy using Tarceva in conjunction with IL- 6 antibodies and JAK inhibitors in mouse models of NSCLC.

A patent application based in part on the work described in the paper and directed to use of IL-6 inhibitors in combination drug therapy for treatment of drug-resistant EGFR mutant NSCLC has been filed by Cold Spring Harbor Laboratory.

Cain, C. SciBX 3(35); doi:10.1038/scibx.2010.1056

Published online Sept. 9, 2010 


\section{Box 1. Targeting IL-6 in glioblastoma.}

A study published in Genes and Development provides additional evidence for IL-6 as a therapeutic target in epidermal growth factor receptor (EGFR) mutant cancers, in particular glioblastoma multiforme (GBM). ${ }^{4}$

About $50 \%$ of GBM cases carry some cells with genomic amplification of wild-type EGFR, and half of these cases also carry activating mutations in EGFR. The presence of both types of cells within these cases of GBM confers a worse prognosis than wild-type EGFR amplification alone and suggests that paracrine signaling from EGFR mutant cells stimulates greater tumor growth.

To understand the basis for this signaling, a group led by Frank Furnari generated GBM tumors carrying a mix of cells overexpressing either wildtype or mutant EGFR. The team found that only a small number of mutant EGFR-expressing cells were required to promote the increased growth of tumor cells expressing amplified levels of wildtype Egfr in the brains of mice.

Furnari is an associate investigator at the Ludwig Institute for Cancer Research Ltd. and an associate professor in the Department of Medicine at the University of California, San Diego.

Furnari, along with postdoctoral fellows Maria-del-Mar Inda and Rudy Bonavia, and Webster Cavenee, professor of medicine and director of the Ludwig Institute branch in San Diego, discovered that the EGFR mutant cells expressed 10-fold greater IL-6 than wild-type cells. In addition, a handful of IL-6-overexpressing cells was sufficient to significantly increase tumor growth in GBM tumors overexpressing wild-type EGFR ( $p=0.0185)$. This suggests that EGFR mutant cells signal through the IL-6 pathway to promote the growth of wild-type EGFR cells.

To test that hypothesis, the team turned to Alnylam Pharmaceuticals Inc., which has developed small interfering RNAs targeting IL-6 and its receptor, gp130. In tumors carrying a mix of siRNA-transfected cells overexpressing wild-type EGFR and mutant EGFR, these siRNAs decreased tumor growth compared with control siRNAs.

Thus, the UCSD team concluded that IL-6 signaling is required to drive tumor growth in mixed tumor populations. IL-6 signals through gp130 to activate Janus kinase (JAK) signaling and phosphorylate signal transducer and activator of transcription 3 (STAT3), a downstream prosurvival transcription factor (see Figure 1, "Targeting Tarceva resistance").

"Future experiments will focus on inhibitors of JAK, which may offer a way to more completely block this signaling pathway than the use of IL-6 antibodies," Furnari told SciBX.

A patent application has been filed regarding parts of the work with the Ludwig Institute and Alnylam as co-owners. -CC

\section{REFERENCES}

1. Yao, Z. et al. Proc. Natl. Acad. Sci. USA; published online Aug. 16, 2010; doi:10.1073/pnas.1009472107

Contact: Raffaella Sordella, Cold Spring Harbor Laboratory, Cold Spring Harbor, N.Y.

e-mail: sordella@cshl.edu

2. Engelman, J.A. \& Janne, P.A. Clin. Cancer Res. 26, 2442-2449 (2007)

3. Gao, S.P. et al. J. Clin. Invest. 117, 3846-3856 (2007)

4. Inda, M.D. et al. Genes Dev.; published online Aug. 15, 2010; doi:10.1101/gad. 1890510

Contact: Frank Furnari, University of California at San Diego, La Jolla, Calif. e-mail: ffurnari@ucsd.edu

5. Iliopoulos, D. et al. Mol. Cell. 39, 493-506 (2010)

6. Seike, M. et al. Proc. Natl. Acad. Sci. USA 106, 12085-12090 (2009)
COMPANIES AND INSTITUTIONS MENTIONED

Alder Biopharmaceuticals Inc., Bothell, Wash.

Alnylam Pharmaceuticals Inc. (NASDAQ:INCY), Cambridge, Mass.

Astellas Pharma Inc. (Tokyo:4503), Tokyo, Japan

AstraZeneca plc (LSE:AZN; NYSE:AZN), London, U.K.

Cold Spring Harbor Laboratory, Cold Spring Harbor, N.Y.

Genentech Inc., South San Francisco, Calif.

Harvard Medical School, Boston, Mass.

Incyte Corp. (NASDAQ:INCY), Wilmington, Del.

Ludwig Institute for Cancer Research Ltd., La Jolla, Calif.

Memorial Sloan-Kettering Cancer Center, New York, N.Y

Roche (SIX:ROG; OTCQX:RHHBY), Basel, Switzerland

University of California, San Diego, La Jolla, Calif. 